\title{
A simple method to measure cell viability in proliferation and cytotoxicity assays
}

\section{Ricardo Carneiro Borra ${ }^{(a)}$ \\ Mônica Andrade Lotufo(a) \\ Sonia Maria Gagioti(a) \\ Fabiana de Mesquita Barros(b) \\ Priscila Maria Andrade ${ }^{(c)}$}

(a) PhD, Professor; (b) MSc, Graduate Student - Graduate Program in Biodentistry, Ibirapuera University (UNIB), São Paulo, SP, Brazil.

(c) Fellow Researcher, Department of Urology (LIM 55), School of Medicine, University of São Paulo (FMUSP), São Paulo, SP, Brazil.

\section{Corresponding author:}

Ricardo Carneiro Borra

Universidade Ibirapuera, Curso de

Odontologia

Av. Interlagos, 1329

São Paulo - SP - Brazil

CEP: 04661-100

E-mail: rcborra@gmail.com

Received for publication on Oct 12, 2008

Accepted for publication on Mar 25, 2009
Abstract: Resazurin dye has been broadly used as indicator of cell viability in several types of assays for evaluation of the biocompatibility of medical and dental materials. Mitochondrial enzymes, as carriers of diaphorase activities, are probably responsible for the transference of electrons from $\mathrm{NADPH}+\mathrm{H}^{+}$to resazurin, which is reduced to resorufin. The level of reduction can be quantified by spectrophotometers since resazurin exhibits an absorption peak at $600 \mathrm{\eta m}$ and resorufin at $570 \mathrm{\eta m}$ wavelengths. However, the requirement of a spectrophotometer and specific filters for the quantification could be a barrier to many laboratories. Digital cameras containing red, green and blue filters, which allow the capture of red (600 to $700 \mathrm{\eta m}$ ) and green (500 to $600 \mathrm{\eta m}$ ) light wavelengths in ranges bordering on resazurin and resorufin absorption bands, could be used as an alternative method for the assessment of resazurin and resorufin concentrations. Thus, our aim was to develop a simple, cheap and precise method based on a digital CCD camera to measure the reduction of resazurin. We compared the capability of the CCD-based method to distinguish different concentrations of L929 and normal $\mathrm{Hu}$ man buccal fibroblast cell lines with that of a conventional microplate reader. The correlation was analyzed through the Pearson coefficient. The results showed a strong association between the measurements of the method developed here and those made with the microplate reader $\left(r^{2}=0.996 ; p<0.01\right)$ and with the cellular concentrations $\left(r^{2}=0.965\right.$; $\mathrm{p}<0.01)$. We concluded that the developed Colorimetric Quantification System based on CCD Images allowed rapid assessment of the cultured cell concentrations with simple equipment at a reduced cost.

Descriptors: Colorimetry / methods; Cells, cultured; Mitochondria / metabolism. 


\section{Introduction}

The biocompatibility of medical and dental materials is routinely evaluated utilizing in vitro methodologies. Cell lines are often cultivated in contact with the target materials, and after a variable period of time, the proliferation (estrogenicity) and death (toxicity) rates are measured. ${ }^{1,2,3}$

Resazurin dye (7-hydroxy-3H-phenoxazin-3-one 10 -oxide) has been broadly used as an indicator of cell viability in several types of proliferation and cytotoxicity assays. ${ }^{4-8}$ The reduction of resazurin correlates with the number of live organisms, such as bacterial, fungi and mammalian cells. Mitochondrial enzymes, as carriers of diaphorase activities, like NADPH dehydrogenase, are probably responsible for the transference of electrons from NADPH $+\mathrm{H}^{+}$ to resazurin, which is reduced to resorufin. ${ }^{9}$

Cellular viability quantification based on resazurin has advantages, including rapidity, reliability, sensitivity, safety and cost. In addition, it keeps cells intact, which permits other parallel analyses, such as mRNA, cytogenetic, apoptosis, and immunophenotyping. ${ }^{6}$ The level of reduction can be quantified by spectrophotometers using appropriate filters, since resazurin exhibits an absorption peak at $600 \mathrm{\eta m}$ and resorufin at $570 \mathrm{\eta m}$ wavelengths. ${ }^{10}$ However, the requirement of a spectrophotometer and specific filters for the quantification of resazurin reduction levels could be a barrier to many laboratories. Digital cameras, containing red, green and blue (RGB) filters, which allow the capture of red (600 to $700 \eta \mathrm{m}$ ) and green (500 to $600 \eta \mathrm{m}$ ) light wavelengths in ranges bordering on resazurin and resorufin absorption bands, could be used as an alternative method for the assessment of resazurin reduction levels. Therefore, using a color digitalized image of microplate load with resazurin and common imageprocessing software it might be possible to isolate red and green intensity values to calculate the level of reduction of resazurin of different samples.

Thus, our aim was to develop a simple, cheap, precise and reliable method based on RGB signals generated by a digital CCD camera to measure the reduction of resazurin dye into resorufin. To test this method, reduction levels of resazurin measured by the digital camera-based system developed were com- pared with those measured by the conventional ELISA microplate reader. Then the viability of the system in quantifying different concentrations of L929 and buccal fibroblast cells in culture was tested.

\section{Material and Methods Reagents}

AlamarBlue $^{\mathrm{TM}}$ was purchased from Biosource (Camarillo, CA, USA, cat.\# DAL1025); resazurin sodium salt (cat.\#R7017) and L-ascorbic acid (cat.\#A5960) were acquired from Sigma (St. Louis, MO, USA). DMEM and RPMI-1640 media supplemented with $2 \mathrm{mM}$ of L-glutamine, $100 \mathrm{U} / \mathrm{mL}$ of penicillin and $100 \mu \mathrm{g} / \mathrm{mL}$ of streptomycin; fetal bovine serum, a solution of Trypsin-EDTA and TPP 96-well tissue culture microplates were purchased from CultiLab (Campinas, SP, Brazil). The resazurin solutions were prepared using phosphate buffered saline from Sigma. L929 cells were purchased from ATCC (Manassas, VA, USA) and a normal Human buccal fibroblast cell line (FLM1) was obtained from the Ibirapuera University cell bank.

\section{Colorimetric Quantification System based on CCD images (CQS)}

A simple system capable of analyzing signals from a 96-well culture microplate was constructed using a negatoscope and a seven mega pixel digital camera (Sony Cybershot, model DSC-P200) positioned on tripod legs $45 \mathrm{~cm}$ above the illuminated surface. In order to measure the well signals, two 24 bit color images of an empty microplate (blank) and of a microplate loaded with samples were captured and analyzed using the software ImageLab (Diracom Bioinformática, São Paulo, SP, Brazil). Based on an area of interest defined manually on the bottom of wells by the user, ImageLab separates the red and green components and measures the density level of each spectrum. The same result can be obtained using the Adobe ${ }^{\circledR}$ Photoshop 7.0 software. Basically, the user must open an image, select the red or green channel, define the area of interest, open the histogram window and search for the mean intensity of the histogram. In contrast to using ImageLab, using Adobe Photoshop 7.0 requires that all of the above steps be performed for each well of each microplate. 
The image from the microplate loaded with samples was used to calculate the density of the red (Wr) and green $(\mathrm{Wg})$ spectra of each well, and an image of an empty microplate was used to calculate the red $(\mathrm{Br})$ and green $(\mathrm{Bg})$ background from the correlated wells. Afterwards, the highest red (MAXBr) and green $(\mathrm{MAXBg})$ background values were determined and the CQS transmittance index (CQSTI) was calculated according to the following formula:

$$
\mathrm{CQSTI}_{\mathrm{mn}}=\mathrm{Wr}_{\mathrm{mn}} \times\left(\mathrm{MAXBr} / \mathrm{Br}_{\mathrm{mn}}\right)-\mathrm{Wg}_{\mathrm{mn}} \times\left(\mathrm{MAXBg} / \mathrm{Bg}_{\mathrm{mn}}\right)
$$

where:

$$
\begin{aligned}
& \mathrm{m}=\text { microplate column } \\
& \mathrm{n}=\text { microplate row } \\
& \mathrm{Wr}_{\mathrm{mn}}=\text { red spectrum density of the well located } \\
& \text { on the } \mathrm{m}^{\text {th }} \text { colum, } \mathrm{n}^{\text {th }} \text { row of a sample } \\
& \text { filled microplate image } \\
& \mathrm{Wg}_{\mathrm{mn}}=\text { green spectrum density of the well } \\
& \text { located on the } \mathrm{m}^{\text {th }} \text { colum, } \mathrm{n}^{\text {th }} \text { row of a } \\
& \text { sample filled microplate image } \\
& \mathrm{Br}_{\mathrm{mn}}=\text { red spectrum density of the well located } \\
& \text { on the } \mathrm{m}^{\text {th }} \text { colum, } \mathrm{n}^{\text {th }} \text { row of an empty } \\
& \text { microplate image } \\
& \mathrm{Bg}_{\mathrm{mn}}=\text { green spectrum density of the well } \\
& \text { located on the } \mathrm{m}^{\text {th }} \text { colum, } \mathrm{n}^{\text {th }} \text { row of an } \\
& \text { empty microplate image } \\
& \mathrm{MAXB}_{\text {red }}=\text { the highest value of } \mathrm{Br} \\
& \mathrm{MAXB}_{\text {green }}=\text { the highest value of } \mathrm{Bg}
\end{aligned}
$$

The final CQSTI is adjusted by discounting the CQSTI mean of the wells used as negative controls (oxidized resazurin).

\section{Evaluation of the CQS method}

After reducing an aliquot of AlamarBlue ${ }^{\mathrm{TM}}$ (Biosource, Camarillo, CA, USA), by autoclaving for 15 minutes and allowing it to cool to room temperature, a curve of reduction was constructed mixing $20 \mu \mathrm{L}$ of eleven different proportions of the oxidized (resazurin: $20,18,16,14,12,10,8,6,4,2$ and $0 \mu \mathrm{L}$ ) and reduced (resorufin: 0, 2, 4, 6, 8, 10, 12, 14, 16, 18 and $20 \mu \mathrm{L}$ ) form of AlamarBlue ${ }^{\mathrm{TM}}$ in $180 \mu \mathrm{L}$ of RPMI-1640 complete medium volume, according to the manufacturer's instructions. Wells in triplicate containing the mixture were digitalized and quanti- fied using the CQS methodology. This experiment was repeated at two different times.

\section{Comparison between AlamarBlue ${ }^{T M}$ and resazurin solutions}

Cell proliferation and cytotoxicity assays are often carried out using a commercial resazurin-based product known as AlamarBlue ${ }^{\mathrm{TM}}$ (Biosource, Camarillo, CA, USA), but a cheaper laboratory-prepared resazurin solution could also be used without compromising rapidity, reliability, sensitivity or safety. ${ }^{7}$ In order to evaluate the sensitivity of both solutions, a colorimetric assay using CQS methodology was performed comparing the reduction curve of AlamarBlue ${ }^{\mathrm{TM}}$ with curves of different concentrations of resazurin solutions. In this case, wells containing $20 \mu \mathrm{L}$ of AlamarBlue ${ }^{\mathrm{TM}}$ or different concentrations of resazurin $(175,350,525$ and $700 \mu \mathrm{M})$ diluted in $180 \mu \mathrm{L}$ of RPMI-1640 complete medium were incubated in triplicate for thirty minutes with different amounts of vitamin $\mathrm{C}\left(0.5 \times 10^{3}, 1.0 \times 10^{3}\right.$, $2.0 \times 10^{3}, \quad 3.0 \times 10^{3}$ and $\left.4.0 \times 10^{3} \mu \mathrm{g} / \mathrm{mL}\right)$, which were used as a reduction agent capable of simulating diaphorase activity. This experiment was repeated at two different times.

\section{Comparison between the CQS method and microplate reader quantification}

With the aim of comparing the reliability and sensitivity of the CQS methodology with data obtained from a spectrophotometer, a curve of reduction constructed using a laboratory-prepared resazurin solution was submitted to a reading by the CQS method and by an ELISA microplate reader. Briefly, wells containing solutions of $180 \mu \mathrm{L}$ of RPMI-1640 and $20 \mu \mathrm{L}$ of the combination of ten different proportions of $700 \mu \mathrm{M}$ oxidized resazurin $(20,18,16,14$, $12,10,8,6,4$ and $0 \mu \mathrm{L}$ ) and of $700 \mu \mathrm{M}$ reduced resazurin $(0,2,4,6,8,10,12,14,16$ and $20 \mu \mathrm{L})$ were analyzed at wavelengths of 595 and $490 \mathrm{~nm}$ using the microplate reader (Bio Tek ELX - 800 - BioTek Instruments, Inc., VT, USA) and compared with measurements performed using the CQS method. Since the autoclaving procedure was not efficient in reducing the resazurin in PBS, the resorufin stock $(700 \mu \mathrm{M})$ was reduced with $500 \mu \mathrm{g} / \mathrm{mL}$ of vitamin 
C. Thus, the correlation of pairs of optical density (OD) and CQSTI values was verified using the Pearson Correlation Statistic Test.

\section{Reliability of the CQS method to quantify different cell densities}

Confluent monolayer adherent L929 and FLM1 cells, grown in RPMI-1640 and DMEM medium respectively, supplemented with $10 \%$ fetal bovine serum, $2 \mathrm{mM}$ of L-glutamine $+100 \mathrm{U} / \mathrm{mL}$ of penicillin / $100 \mu \mathrm{g} / \mathrm{mL}$ of streptomycin, and maintained at $37^{\circ} \mathrm{C}$ in a $5 \% \mathrm{CO}_{2}$ atmosphere, were trypsinized and resuspended at a concentration of $10^{6}$ cells $/ \mathrm{mL}$. Different cell concentrations of L929 and FLM1 cells diluted in $180 \mu \mathrm{L}$ of RPMI-1640 and DMEM complete medium respectively were seeded in a 96-well tissue culture microplate and incubated in triplicate (FLM1) and quadruplicate (L929) with AlamarBlue $^{\mathrm{TM}}$ and resazurin $(700 \mu \mathrm{M})$ for up to 35 hours. Images were taken and analyzed at different time points using the CQS methodology during the entire period of incubation.

\section{Statistical analysis}

To determine the level of correlation between AlamarBlue $^{\mathrm{TM}}$ / resazurin and CQSTI and the correlation between the optical density (OD) of the microplate reader with the CQSTI, the values were plotted in a scatter plot and the Pearson Correlation was calculated using SPSS 10.0 software (SPSS Inc. Chicago, IL, USA). $\mathrm{p}<0.05$ was considered significant.

\section{Results}

\section{Evaluation of the CQS method}

The CQSTI was strongly correlated with the intensity of the AlamarBlue ${ }^{\mathrm{TM}}$ reduction level. This correlation was linear until the mixture of $9 \%$ of resorufin $+1 \%$ of resazurin $\left(y=1.64 x+0.45 ; r^{2}=0.99\right.$; $\mathrm{p}<0.01$ ) (Graph 1). The densities of red and green spectra correlated directly with the level of resorufin and resazurin respectively, in a dose dependent manner, while the density of the blue did not (Figure 1).
Graph 1 - Transmittance index of AlamarBlue ${ }^{\text {tw }}$ calculated from the digitalized image of triplicate wells containing $20 \mu \mathrm{L}$ of different proportions of oxidized (blue) and reduced (pink) AlamarBlue "ituted in $180 \mu \mathrm{L}$ of RPMI1640 complete medium. A closed circle

$(\bullet)$ represents samples whose signal was not saturated and an open circle (o), a saturated signal. The line shows the relation between concentrations of reduced AlamarBlue "rw (resorufin) and the CQSTI. Equation regression: $y=1.64 x+0.45 ; r^{2}=0.99$ and $p<0.01$.

Figure 1 - Image of the three separated spectra ( $R, G$ and $B)$ from wells containing $20 \mu \mathrm{L}$ of different concentrations of resazurin (oxidized) and resorufin (reduced) diluted with $180 \mu \mathrm{L}$ of RPMI-1640 complete medium. The level of resazurin reduction in each spectrum is shown as a gray tone (range 0 to 255).

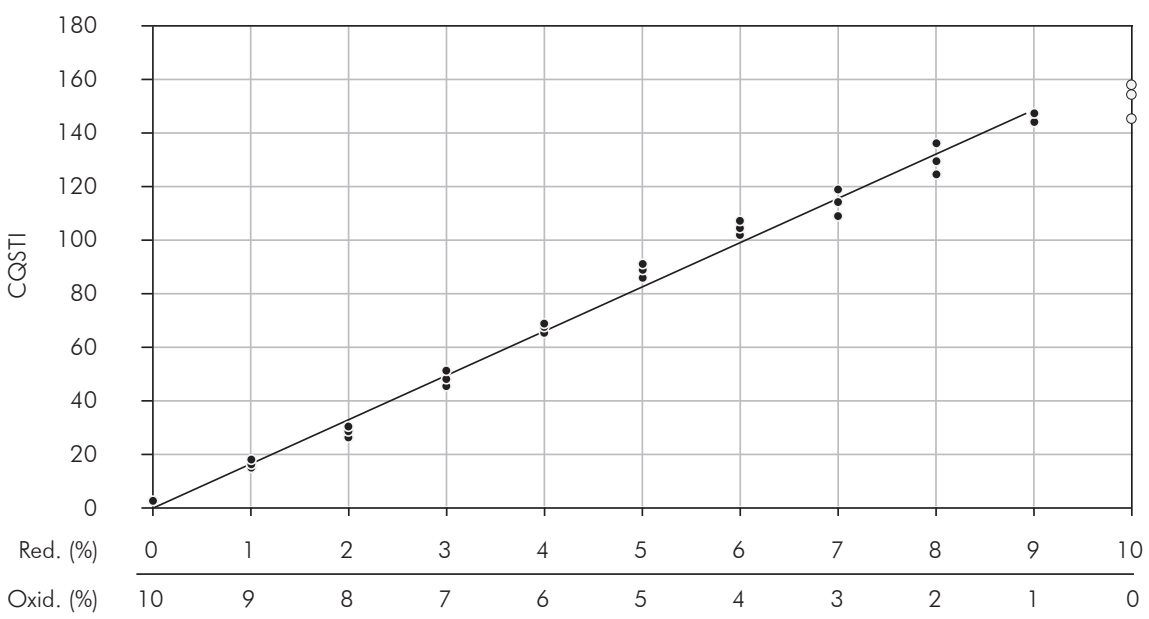

$\mathbf{R}$

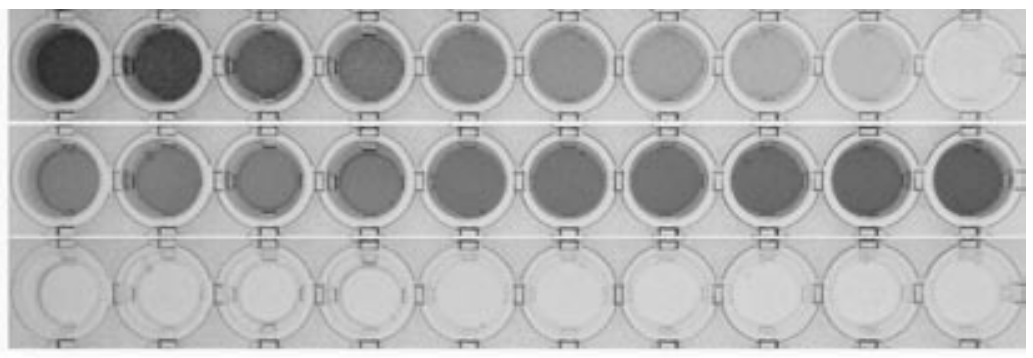

$\begin{array}{lllllllllll}\text { Red. }(\mu M) & 0 & 14 & 21 & 28 & 35 & 42 & 49 & 56 & 63 & 70 \\ \text { Oxid. }(\mu M) & 70 & 63 & 56 & 49 & 42 & 35 & 28 & 21 & 14 & 0\end{array}$ 


\section{Comparison between AlamarBlue ${ }^{\mathrm{TM}}$ and resazurin solutions}

Vitamin C reduced both resazurin and AlamarBlue proportionally to its concentration. Resazurin at a concentration of $525 \mu \mathrm{M}$ was more closely associated with the AlamarBlue reduction curve (Graph 2). The equivalent concentration of resazurin related to AlamarBlue was calculated as $560 \mu \mathrm{M}$ (data not shown).

\section{Comparison between the CQS method and microplate reader quantification}

The concentration of resazurin measured by the CQS was strongly correlated with the values measured by the ELISA microplate reader ( $\mathrm{y}=206.8 \mathrm{x}$; $r^{2}=1.0$ and $\left.p<0.01\right)($ Graph 3).

\section{Reliability of the CQS method to quantify different cell densities}

Graph 4 shows that the numbers of L929 and FLM1 cells were strongly correlated with values calculated using the CQS method (CQSTI). The time of incubation and the number of cells influenced directly the level of reduction of both resazurin $700 \mu \mathrm{M}$ and AlamarBlue. The results showed that the best incubation time range for quantifying FLM1 was

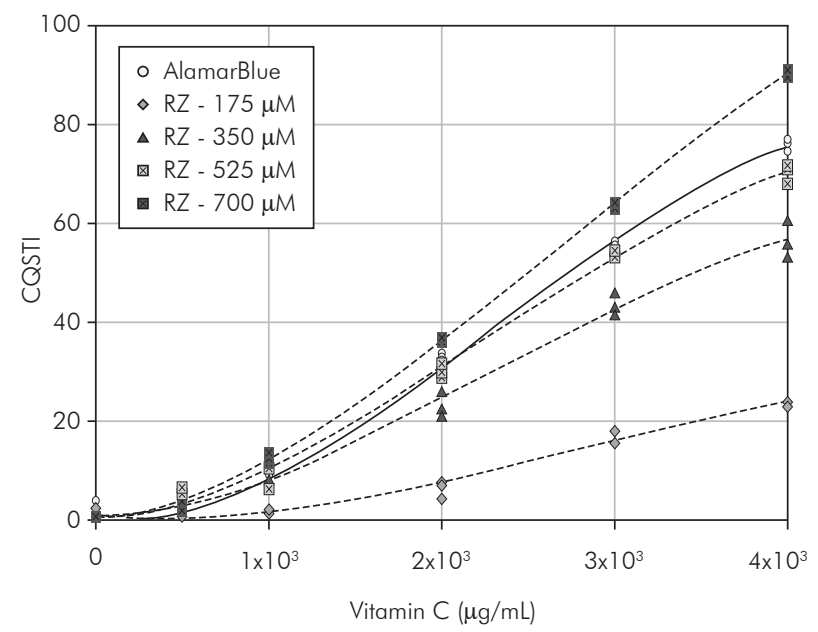

Graph 2 - Relation between AlamarBlue and resazurin (RZ) curves as a function of vitamin $C$ concentration. Transmittance index calculated from the digitalized image of triplicate wells containing $20 \mu \mathrm{L}$ of AlamarBlue and different concentrations of resazurin diluted in $180 \mu \mathrm{L}$ of RPMI-1640 complete medium with different amounts of vitamin $\mathrm{C}$.
11 to 19 hours; for quantifying L929, a murine tumor cell line, the best incubation time range was 6 to 10 hours. The sensitivity of the method was superior when $700 \mu \mathrm{M}$ of the resazurin solution were employed for quantification of the concentrations of both cell types. The $700 \mu \mathrm{M}$ solution of resazurin reached the saturation point around a CQSTI of 160, contrasting with AlamarBlue, which reached that point around a CQSTI of 120 (Graph 4).

\section{Discussion}

Resazurin dye (7-hydroxy-3H-phenoxazin-3-one 10 -oxide) has been broadly used as a reliable indicator of cell viability in proliferation and cytotoxicity assays. ${ }^{4-13}$ Analogous to resazurin, the dye 3[4,5-dimethylthiazol-2-yl]-2,5-diphenyl tetrazolium bromide (MTT) has been also employed to quantify cell viability; however, the resazurin assay seemed to be slightly more sensitive than the MTT assay. ${ }^{9,12}$ Resazurin is probably a better redox indicator for cell viability because it does not interfere with the reactions of the electron transport chain since the midpoint redox potential of resazurin is greater than that of any of the components of the electron transport chain while indicators with an inferior redox potential such as MTT cannot detect the reduction

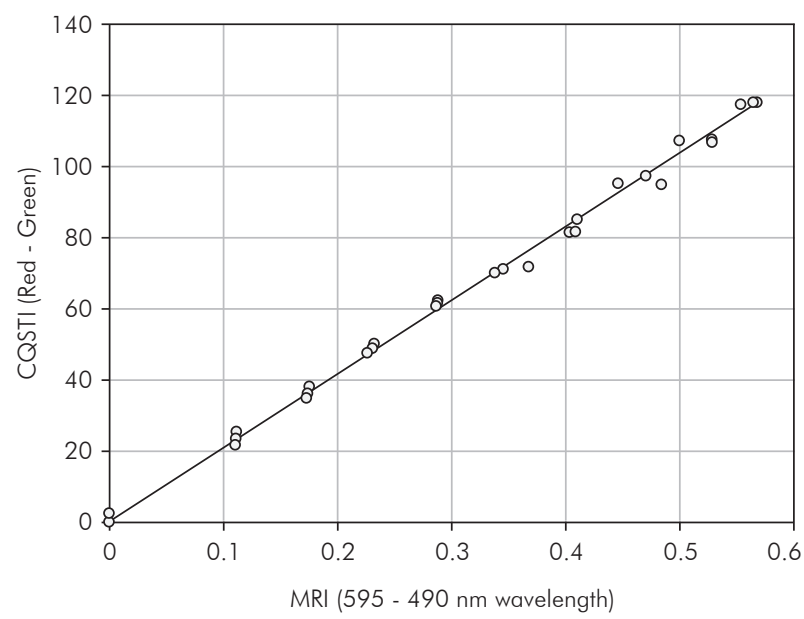

Graph 3 - Comparison between the transmittance indexes obtained by the CQS and microplate reader methods. Triplicate wells containing $20 \mu \mathrm{L}$ of ten different concentrations of resazurin and resorufin diluted with $180 \mu \mathrm{L}$ of RPMI-1640 complete medium, read using the CQS and the microplate reader. Equation regression: $y=206.8 x ; r^{2}=1.0$ and $p<0.01$. MRI: Microplate reader index. 

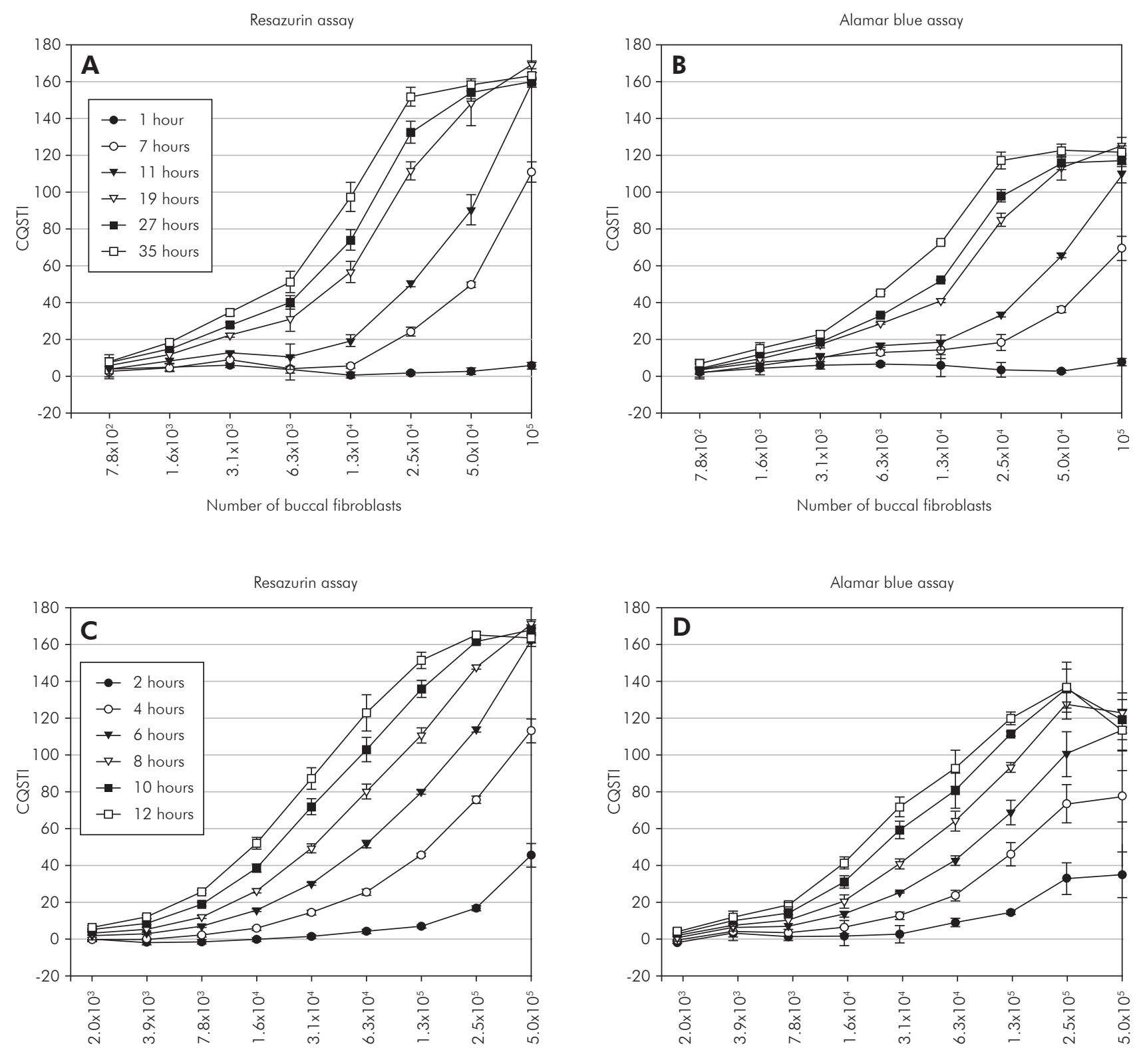

Number of $\mathrm{L} 929$ cells

Number of $L 929$ cells

Graph 4 - Comparison of profiles of reduction of different concentrations of L929 and FLM1 cells assessed by AlamarBlue and $700 \mu \mathrm{M}$ resazurin solution dyes. The CQSTI was represented by mean \pm standard deviation.

mediated by cytochromes. ${ }^{13}$ After comparing the sensitivity of six assays for quantification of the biofilms formed by a broad range of microorganisms in 96-well microtiter plates, the resazurin-based assay has proven to be one of the best alternatives. ${ }^{14}$

However, in order to quantify resazurin reduction, a spectrophotometer and specific filters at 600 and $570 \eta \mathrm{m}$ are required. The use of an RGB CCD digital camera could be an alternative method, since it contains filters that work between 600 to $700 \mathrm{\eta m}$ (red) and 500 to $600 \mathrm{\eta m}$ (green). In this case, a simple inexpensive methodology capable of capturing and analyzing images from a microplate, denominated Colorimetric Quantification based on CCD Images (CQS), was developed and tested regarding its ability to monitor and quantify cell viability.

Quantification was based on assessment of the amount of red and green light emanating from the 
resazurin samples (transmittance). In order to obtain the intensity of red and green light, the microplate was digitalized, and each component $\mathrm{R}$ and $\mathrm{G}$ from the color image was separated using the ImageLab software. Next, the density of each component corresponding to the bottom well area was determined and used to create the transmittance index.

The normalization procedures based on background reference and the use of the difference between the red and green spectra as a mean parameter in the formula was able to neutralize systematic errors related to the parallax effect and non-perfect homogeneity of illumination. This was possible because the difference between the red and green components remains constant regardless of the intensity of light that passes through the wells (data not shown). The use of a scanner with a transparency adaptor could be another alternative. In 2002, Gabrielson et al. ${ }^{11}$ evaluated the use of AlamarBlue indicators for measuring relative growth of microorganisms in microplate using a scanner-digitalized image. Nevertheless, since they did not separate the color components, the difference of reduction level was not detectable, although visible to the naked eye.

The relationship between the CQSTI and the resazurin / resorufin standard curve showed a strong linear association, indicating that the index could be useful for measuring the reduction level of resazurin and, indirectly, cell viability. Furthermore, the results obtained using a microplate reader were strongly correlated with the CQS method.

Although cell viability is often assessed using

\section{References}

1. Oh SH, Choi SY, Choi SH, Lee YK, Kim KN. The influence of lithium fluoride on in vitro biocompatibility and bioactivity of calcium aluminate-pMMA composite cement. J Mater Sci Mater Med. 2004 Jan;15(1):25-33.

2. Taira M, Toguchi MS, Hamada Y, Takahashi J, Itou R, Toyosawa $\mathrm{S}$ et al. Studies on cytotoxic effect of nickel ions on three cultured fibroblasts. J Mater Sci Mater Med. 2001 May;12(5):373-6.

3. Moretti Neto RT, Mello I, Moretti AB, Robazza CR, Pereira AA. In vivo qualitative analysis of the biocompatibility of a commercial product known as AlamarBlue ${ }^{\mathrm{TM}}$, a laboratory-prepared resazurin solution could be employed as a cheaper alternative. Analysis of the performance of AlamarBlue ${ }^{\mathrm{TM}}$ and different concentrations of the resazurin solution demonstrated that a resazurin concentration of $560 \mu \mathrm{M}$ produced an identical curve in a vitamin $\mathrm{C}$ reduction assay. Moreover, the assay based on a $700 \mu \mathrm{M}$ resazurin solution was more sensitive than that based on AlamarBlue ${ }^{\mathrm{TM}}$.

In order to test the usefulness of the method, buccal fibroblast FLM1 and L929 cells were incubated in triplicate and quadruplicate, respectively, with $700 \mu \mathrm{M}$ of resazurin solution and AlamarBlue ${ }^{\mathrm{TM}}$ for 35 hours. The results showed a strong correlation between the CQSTI and the number of cells regardless of the type of solution employed. The CQSTI increased in a time- and cell density-dependent manner until a saturation point. The correlation between cell number and time of incubation was more stable and had a higher amplitude when the $700 \mu \mathrm{M}$ resazurin solution was used. The method was capable of distinguishing narrow variations in the number of cells between $7 \times 10^{2}$ up to $5 \times 10^{5}$ cells, principally after 11 hours of incubation for FLM1 and 6 hours for L929 cells.

\section{Conclusions}

It was concluded that the Colorimetric Quantification System based on CCD Images allowed rapid assessment of many cultured cell samples with simple equipment at a reduced cost, thus offering many laboratories a cost-effective alternative to the currently used methods. different cyanoacrylate-based adhesives. Braz Oral Res. 2008 Jan-Mar;22(1):43-7.

4. Ahmed SA, Gogal RM Jr, Walsh JE. A new rapid and simple non-radioactive assay to monitor and determine the proliferation of lymphocytes: an alternative to $[3 \mathrm{H}]$ thymidine incorporation assay. J Immunol Methods. 1994 Apr 15;170(2):21124.

5. Andrade PM, Chade DC, Borra RC, Nascimento IP, Villanova FE, Leite LC et al. The therapeutic potential of recombinant BCG expressing the antigen S1PT in the intravesical treatment 
of bladder cancer. Urol Oncol. 2009 Mar 7. [in press] [Epub ahead of print]

6. Zhi-Jun Y, Sriranganathan N, Vaught T, Arastu SK, Ahmed SA. A dye-based lymphocyte proliferation assay that permits multiple immunological analyses: mRNA, cytogenetic, apoptosis, and immunophenotyping studies. J Immunol Methods. 1997 Dec 15;210(1):25-39.

7. Nakayama GR, Caton MC, Nova MP, Parandoosh Z. Assessment of the Alamar Blue assay for cellular growth and viability in vitro. J Immunol Methods. 1997 May 26;204(2):205-8.

8. Nociari MM, Shalev A, Benias P, Russo C. A novel one-step, highly sensitive fluorometric assay to evaluate cell-mediated cytotoxicity. J Immunol Methods. 1998 Apr 15;213(2):15767.

9. O’Brien J, Wilson I, Orton T, Pognan F. Investigation of the Alamar Blue (resazurin) fluorescent dye for the assessment of mammalian cell cytotoxicity. Eur J Biochem. 2000 Sep;267(17):5421-6.
10. Zalata AA, Lammertijn N, Christophe A, Comhaire FH. The correlates and alleged biochemical background of the resazurin reduction test in semen. Int J Androl. 1998 Oct;21(5):28994.

11. Gabrielson J, Hart M, Jarelöv A, Kühn I, McKenzie D, Möllby R. Evaluation of redox indicators and the use of digital scanners and spectrophotometer for quantification of microbial growth in microplates. J Microbiol Methods. 2002 Jun;50(1):63-73.

12. Hamid R, Rotshteyn Y, Rabadi L, Parikh R, Bullock P. Comparison of alamar blue and MTT assays for high through-put screening. Toxicol In Vitro. 2004 Oct;18(5):703-10.

13. Petrenko YA, Gorokhova NA, Tkachova EN, Petrenko AY. The reduction of Alamar Blue by peripheral blood lymphocytes and isolated mitochondria. Ukr Biokhim Zh. 2005 SepOct; $77(5): 100-5$.

14. Peeters E, Nelis HJ, Coenye T. Comparison of multiple methods for quantification of microbial biofilms grown in microtiter plates. J Microbiol Methods. 2008 Feb;72(2):157-65. 